\title{
Identification of adverse drug-drug interactions through causal association rule discovery from spontaneous adverse event reports
}

\author{
Ruichu Cai ${ }^{1, \dagger},{ }^{*}$, Mei Liu ${ }^{2,+,}$, , Yong Hu${ }^{3, \dagger}$, Brittany L. Melton ${ }^{4}$, Michael E. Matheny ${ }^{5,6}$, \\ Hua $\mathbf{X u}^{7}$, Lian Duan ${ }^{8}$, Lemuel R. Waitman ${ }^{2}$
}

\begin{abstract}
Objective: Drug-drug interaction (DDI) is of serious concern, causing over $30 \%$ of all adverse drug reactions and resulting in significant morbidity and mortality. Early discovery of adverse $\mathrm{DDI}$ is critical to prevent patient harm. Spontaneous reporting systems have been a major resource for drug safety surveillance that routinely collects adverse event reports from patients and healthcare professionals. In this study, we present a novel approach to discover DDIs from the Food and Drug Administration's adverse event reporting system.

Methods: Data-driven discovery of DDI is an extremely challenging task because higher-order associations require analysis of all combinations of drugs and adverse events and accurate estimate of the relationships between drug combinations and adverse event require cause-andeffect inference. To efficiently identify causal relationships, we introduce the causal concept into association rule mining by developing a method called Causal Association Rule Discovery (CARD). The properties of V-structures in Bayesian Networks are utilized in the search for
\end{abstract}


causal associations. To demonstrate feasibility, CARD is compared to the traditional association rule mining (AR) method in DDI identification.

Results: Based on physician evaluation of 100 randomly selected higher-order associations generated by CARD and AR, CARD is demonstrated to be more accurate in identifying known drug interactions compared to AR, $20 \%$ vs. $10 \%$ respectively. Moreover, CARD yielded a lower number of drug combinations that are unknown to interact, i.e., 50\% for CARD and $79 \%$ for AR. Conclusion: Evaluation analysis demonstrated that CARD is more likely to identify true causal drug variables and associations to adverse event.

Keywords: Drug-drug interaction; Adverse Drug Reaction; Causality; Association Rule

\section{Introduction}

Each time a person uses a prescription medication, there is a potential for an adverse drug reaction (ADR). That potential increases with an increase in the number of concurrent medications being used. Between 2009-2012, about $47 \%$ of the United States population reported they had used at least one prescription medication in the past 30 days and almost $11 \%$ reported using at least five prescription medications in the same period. This equated to approximately $\$ 270$ billion spent on prescription medications in 2013 [1]. ADRs have been attributed to cause over 770,000 injuries and 100,000 deaths each year in US [2], resulting in an annual cost of more than $\$ 136$ billion [3]. Studies have estimated that drug-drug interactions (DDIs) may account for up to $30 \%$ of all unexpected adverse drug reactions (ADRs) [4].

Adverse DDls can be preventable if discovered early. Unfortunately, it is extremely difficult to study DDIs before market approval. During premarketing surveillance, new drugs can only be tested for interactions with existing drugs using in vivo and in vitro methods [5]. However, drugs can interact in many different ways [6], it is infeasible to examine every possible type of interaction for all drug combinations [7]. Additionally, many DDIs require certain amount of exposure to manifest and rare DDls may take several exposures to occur [8]. Therefore, postmarketing surveillance becomes necessary for the early detection of unexpected adverse DDIs in the general population.

To facilitate postmarketing drug safety surveillance, the United States Food and Drug Administration (FDA) established the FDA Adverse Event Reporting System (FAERS) to collect ADR reports from healthcare professionals, patients, and pharmaceutical manufacturers [9]. Similarly at international level, the World Health Organization (WHO) is maintaining a large database of ADR reports, VigiBase [10]. These data provide a significant opportunity to study ADRs computationally. Numerous signal detection algorithms were designed for identifying relationships between drugs and adverse events (AEs) in spontaneous reports. Despite the number of existing algorithms, all have drawbacks that limit their effectiveness, such as noisy 
results with diminishing accuracy and low robustness due to low signal-to-noise ratio, high dimensionality of the data, and limited sample sizes. The algorithms are largely based on the statistical disproportionality theory such as relative reporting ratio $(R R)$ to quantify the degree of unexpectedness of a relationship $[9,11,12]$. Both the FDA and WHO utilize adjusted versions of RR for flagging potential ADRs $[13,14]$. These algorithms primarily focus on binary relationships consisting of one drug and one AE, e.g., cerivastatin $\rightarrow$ muscle injury $[15,16,17,18]$.

To find higher-order relationships raised by DDls, e.g., aspirin + warfarin $\rightarrow$ bleeding, methods have been developed and evaluated on subsets of drugs and specific AEs in the spontaneous reporting systems [19,20,21]. For instance, van Puijenbroek et al. used logistic regression analysis to examine the influence of combined use of itraconazole and oral contraceptives on delayed withdrawal bleeding [19] and diuretics and non-steroidal antiinflammatory drugs on symptoms indicating decreased efficacy of diuretics [21]. Thakrar et al. [20] investigated two statistical models in detecting four known DDls and Tatonetti et al. [22,23] built profiles for eight clinically important AEs based on side effects of drugs known to produce them and predicted potential interactions by searching for drug pairs that match the profiles. Furthermore, Harpaz et al. [24,25] employed association rule mining and bi-clustering algorithms to infer associations between drug combinations and adverse event.

Among the existing pharmacovigilance studies, associations are the most studied relationships. However, an association does not necessarily imply causality [26]. Causal relationships do not only indicate two variables are related, but also how they are related and interacted. One step further, the causal mechanism ensures changes in causal variable directly caused changes in the effect variable. For example, we do not only want to know a particular drug is associated with renal failure, but also we want to know definitively whether the association is due to an adverse reaction or a disease. Without knowing the true relationship, plain associations can lead to false conclusions, e.g., the drug causes renal failure.

Causality is at the center stage of biomedical research and work on how to identify it has primarily taken a pragmatic approach with randomized controlled trials (RCTs) being treated as the gold standard for causal inference; however RCT methods have many well-known limitations [27,28,29]. Experiments are also frequently infeasible due to legal, ethical and practical constraints - no one would randomly assign individuals to smoke to assess its health risks. Instead, large-scale observational datasets on a population can be an indispensable resource for causal inference. Despite significant advances in causal discovery theory that have enabled the computational modeling and learning of causal structures from data $[30,31,32,33]$, application of causal discovery algorithms is seriously hindered by its high computational cost [34] and its strict causal mechanism assumption [31,32]. In addressing the challenge, researchers have proposed constraint-based causal discovery methods, instead of searching 
for a complete Bayesian network they aim to learn local casual structures such as the Markov blanket [35,36,37], V-structures [38] , causal cut [39]. As association rule mining (AR) has been demonstrated to be versatile in exploring relationships in large datasets, researchers have attempted to take advantage of AR for causal discovery by manipulating observational data as in retrospective cohort studies [40,41].

In this study, we aim to investigate whether it is feasible to augment AR to discover causal rules for DDI identification. Our study differs from prior related studies in the following aspects: (1) we propose a Causal Association Rule Discovery (CARD) method by exploiting the properties of $\mathrm{V}$-structures in $\mathrm{CBN}$ to guide the causal association rule search; (2) to our knowledge, this is the first CBN-based causal discovery framework for identifying DDIs and their causal relationships to adverse events.

\section{Materials and Methods}

Preparation of datasets. We collected a large sample of spontaneous reports published between the years of 2004 to 2012 from FAERS that are categorized as having a "serious" patient outcome. In other words, only reports with outcome codes listed in Table 1 are included in the study. Additionally, we restricted our mining process to reports with mentions of at least two drugs; focusing our study on detecting adverse effects corresponding to drug combinations. Furthermore, we excluded duplicated reports and limited the analysis to drugs and AEs that occurred in at least five "serious" reports. The overall process is depicted in Figure 1 and following sections describe each key step in detail. 


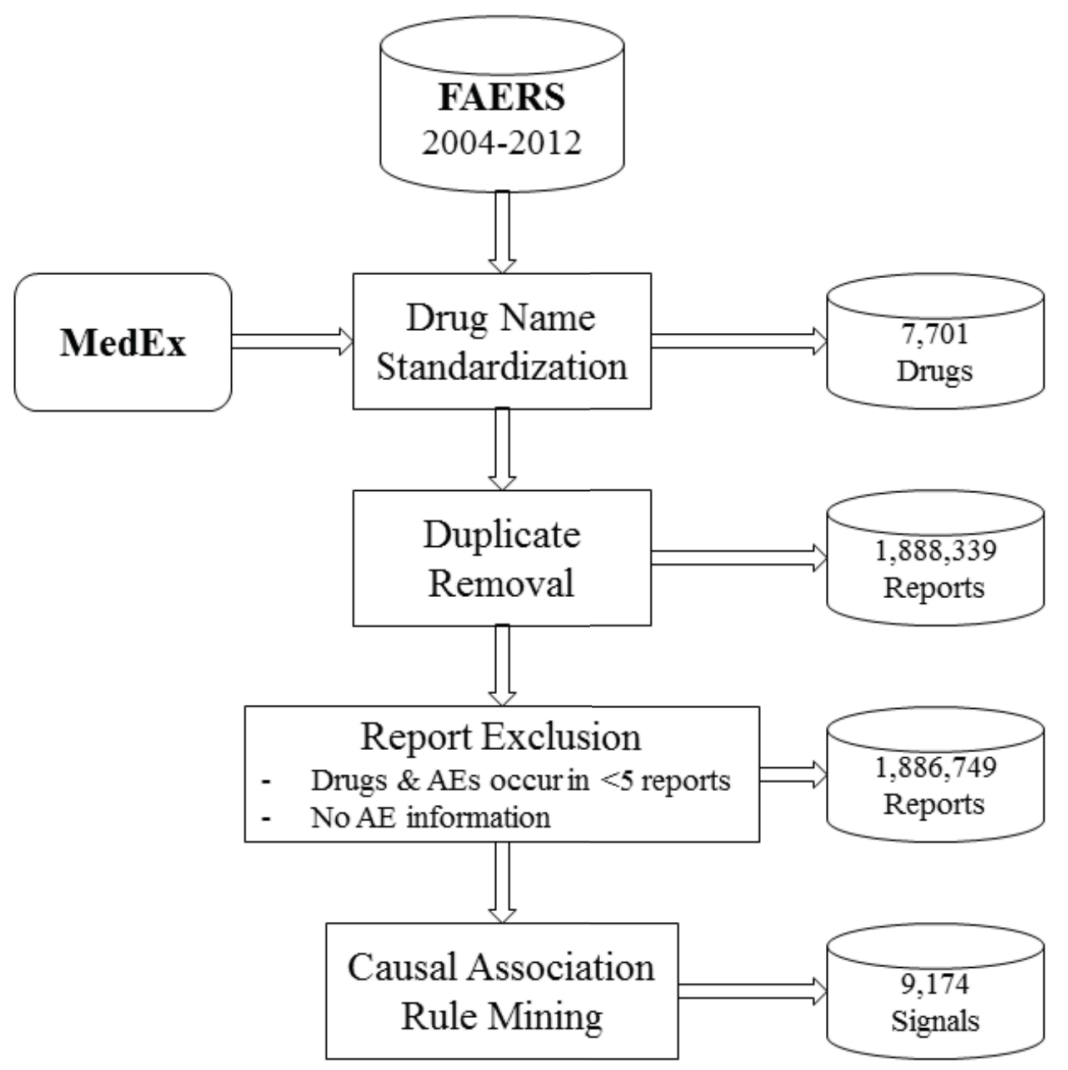

Figure 1. Overview of the mining process

Table 1. Serious outcome code of FAERS reports utilized in this study

\begin{tabular}{|l|l|}
\hline Outcome Code & Description \\
\hline DE & Death \\
\hline LT & Life-threatening \\
\hline HO & Hospitalization - initial or prolonged \\
\hline DS & Disability \\
\hline CA & Congenital Anomaly \\
\hline RI & Required intervention to prevent permanent impairment/damage \\
\hline
\end{tabular}

Entity standardization. Drug names in FAERS are entered as free-text in a variety of forms. For example, the antidiabetic drug metformin can be entered as Fortamet, Diaben/metformin, Diabex metformin hydrochloride, and etc. The terms can also contain dose or route information, 
e.g., "500mg metformin", and active ingredient information, e.g., "Competact (pioglitaxone / metformin hydrochloride)". To codify the medications, we used MedEx [42] to extract drug names out of the free-text terms and mapped each to a generic drug concept in RxNorm. When textual terms contain multiple active ingredients, the mapping was done separately for individual ingredient. Drug names that could not be mapped were still included in the analysis in their original form. Drug names can also be entered as a specific drug or a drug class, for example, aspirin vs. analgesics. Although aspirin falls under the analgesic class, we did not attempt to make the translation, so they are included as separate entities. Since adverse outcome reported in FAERS are already coded using the MedDRA terminology [43] (a terminology developed for adverse drug event applications), we treated each coded $A E$ as a unique entity.

Duplicate report removal. Studies [44,45] have suggested that FAERS may contain $5 \%$ to $20 \%$ duplicated reports, introducing sample bias that may result in spurious signals. FDA utilizes a proprietary algorithm to remove duplicates for analysis. As an alternative, other studies have instead searched for reports with at least eight drugs or AEs and determined reports as duplicate if they match on all the reported drugs, AEs, and patient demographic information $[24,25]$. Setting the threshold at eight is to minimize the probability of a match by chance. Our study adopted this approach in removing duplicated reports.

Dataset statistics. Statistics on FAERS reports published between 2004 and 2012 are shown in Table 2. The full set contains 4,509,229 reports. After excluding reports with non-serious outcome and duplicates, only $41.88 \%$ of the original set remained. Additionally, we removed reports that have no $A E$ information and those only contain drugs and $A E s$ appearing in less than 5 reports, reducing the sample size to 1,886,749 reports; covering 7,701 unique generic drug names (reduced from 351,980 unique textual entries) and 11,569 MedDRA coded AEs.

Table 2. FAERS data statistics by year

\begin{tabular}{|l|l|l|l|}
\hline Year & Reports & Reports with serious outcome & Reports after duplicate removal \\
\hline 2004 & 272,295 & 141,989 & 135,463 \\
\hline 2005 & 325,674 & 164,509 & 157,439 \\
\hline 2006 & 323,791 & 168,401 & 160,793 \\
\hline 2007 & 378,176 & 169,171 & 157,759 \\
\hline 2008 & 441,009 & 198,114 & 180,244 \\
\hline 2009 & 491,305 & 234,268 & 209,854 \\
\hline 2010 & 673,170 & 285,939 & 253,287 \\
\hline
\end{tabular}




\begin{tabular}{|l|l|l|l|}
\hline 2011 & 782,795 & 357,499 & 313,684 \\
\hline 2012 & 821,014 & 352,888 & 319,816 \\
\hline Total & $4,509,229$ & $2,072,778$ & $1,888,339$ \\
\hline
\end{tabular}

Association rule mining (AR). Association rule mining is a well-established data mining method for discovering relationships in data and its algorithmic variations have been developed for ADR detection $[24,40,46]$. An association rule is an implication expression of the form $A \rightarrow B$, where $A$ and $B$ are two event sets that do not share any common events. In the case of ADR detection, $A$ denotes a set of drugs and $B$ denotes a set of AEs, e.g., $A=$ (cerivastatin) $\rightarrow B=$ (muscle injury). An event set can contain one or more items, multi-item associations. For example, $A=$ (aspirin + warfarin) $\rightarrow B=$ (Bleeding) indicates taking aspirin and warfarin together is associated with the bleeding.

To assess the strength of an association rule, the best-known measures are support and confidence. Support of an association rule $S(A \rightarrow B)$ is the proportion of records in which $A$ and $B$ both appear. Confidence of an association rule $C(A \rightarrow B)$ is the probability $P(B \mid A)$ of finding the consequent $B$ given the antecedent $A$. Therefore, support measures the unexpectedness of the rule and confidence measures the reliability of the rule. These two measures allow the screening of interesting rules from a set of all possible rules. An interesting association rule is required to satisfy user-specified minimum thresholds on support and confidence at the same time. The AR method described in Harpaz et al. [24] is implemented in this study as the baseline algorithm for comparison and the relative reporting ratio (RR) is used accordingly as the proxy for measuring rule strength rather than the traditional confidence.

Causal association rule discovery (CARD). As associations may not indicate causal relationships, our study aims to detect true causal relationships between drug combinations (drug-drug interactions) and adverse events by proposing a new method called causal association rule discovery (CARD). Given a set of spontaneous reports in the FAERS database, , let be the binary indicators of the drugs taken, where represents whether a patient has taken the th drug or not. Let be a binary indicator of the occurrence of an AE and represents an ADR report $j$. Let denote the event that th drug is taken () and denote the event that an $A E$ has occurred (), then an AE triggered by a drug-drug interaction (DDI) can be formulated as an association rule, . As the study focuses on DDIs, only rules with are considered. To formalize the concept of causal association rules, we derive important properties from Causal Bayesian Network (CBN) and develop an efficient search method. 
In the simplest DDI case, a CBN of three variables with being the possible causes and $y$ being the effect has four primitive local structures as shown in Figure 2. Figure $2 a, 2 b$, and $2 c$ are independent-equivalent BNs because they entail the same set of conditional independence relationships. In other words, variable is conditionally independent of variable given variable (i.e. ). However, Figure 2d implies a different assertion that is conditionally dependent of given $y$, referred to as the $\mathrm{V}$-Structure in $\mathrm{BN}$, which is well known and studied in inductive causation methods [26].

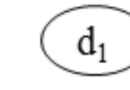

omeprazole
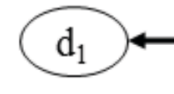

vancomycin

aspirin

aspirin
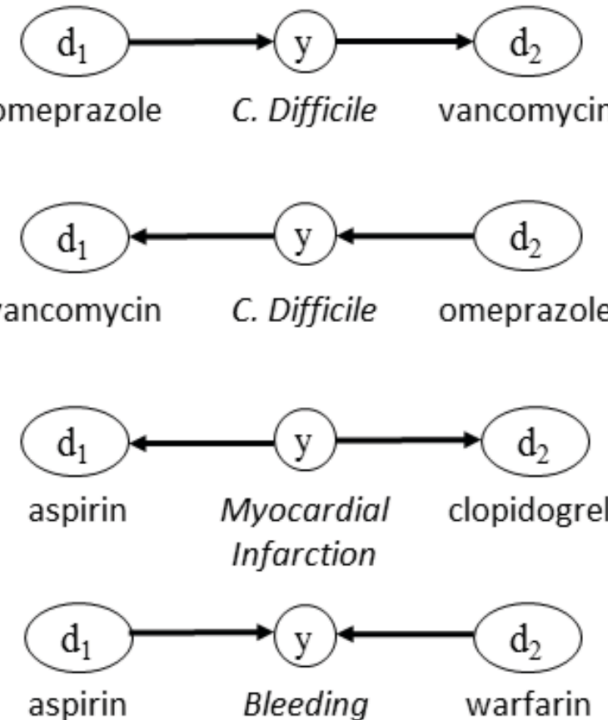

C. Difficile

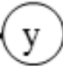

C. Difficile
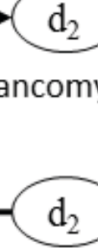

vancomycin

omeprazole (c)

Figure 1. Basic BN structures: (a-c) conditional independence equivalent; (d) V-structure

In contrast to other CBN local structures, V-structure is more robust and discriminating in causality identification problems because it is not statistically equivalent to any other structures involving the same variables. It is the only local structure that can be used to confirm the direction of causal relationships. If a true $\mathrm{V}$-structure forms as in Figure $2 \mathrm{~d}$, the two drugs involved must be interacting to cause $A E$. From the statistical aspect, the following statistical independence relation holds for all the V-structures, 1) holds and 2) does not hold, where . Based on the above essential properties of $\mathrm{V}$-structure, we derived the following causal association interesting measure (CAIM) for .

Here, denotes the normalized mutual information [47]. A high CAIM score indicates that both, are highly associated with $y$, i.e. the role of , and previously non-associated, became highly associated given $y$, i.e. the role of . 
For the rule with more than three antecedents, any sub-rules containing two antecedents must also form a V-structure with the adverse event, . Because the interestingness of rule is dependent on the weakness of its sub-rules, the generalized interestingness measure is defined as follows.

To efficiently search through the large space of all possible rules and estimate interestingness of the rules, we derived a pruning strategy based on properties of V-structures and an incremental updating strategy for CAIM. Algorithm details are available in Supplementary Appendix.

\section{Results}

Performance evaluation. Following common practice in pharmacovigilance studies in evaluating clinical validity of DDIs [15,16,24], we randomly selected 100 rules identified by CARD and presented them to a pharmacist for manual review. The pharmacist used both Micromedex and Epocrates [48,49] as the clinical reference and settled any disagreements by checking with UpToDate [50]. The pharmacist and a physician also helped us in characterizing the identified rules with a taxonomy developed based on observations. For algorithm performance comparison, we implemented the AR method as presented in Harpaz et al. [24] that was shown to be effective in identifying higher-order associations, and performed the same analysis on its 100 randomly selected findings.

Higher-order drug-event relationship identification. For the baseline AR algorithm, we aligned this study with the study in [24] by setting AR thresholds at minimum support of 100 and $\mathrm{RR}$ of 2 . The minimum support and $\mathrm{RR}$ thresholds were observed to be a balancing point between the number of rules generated and variation in content (e.g., drugs and AEs in the rules). Low thresholds will result in large set of rules with many false positives. High thresholds, on the other hand, will result in less variation in content. Using the proposed thresholds, AR in total produced 424 higher-order association rules with combinations containing at least 2 drugs and among them 57 contained 3 or more drugs. Analogously, CARD also used minimum support of 100 and the CAIM score threshold was set at $\geq 0.04$ to produce similar number of rules (i.e., 457) and among which 10 contained 3 or more drugs (e.g., an identified combination included doxorubicin hydrochloride, prednisone, and rituximab).

From the identified drug-event relationships, we randomly selected 100 for validation and developed a taxonomy from the analysis. The taxonomy characterizing drugs and associations along with the proportions of each category in the 100 randomly sampled findings 
is presented in Table 3. It contains observed proportions for both AR and CARD. Table 4 provides the taxonomy that characterizes the rules along with its proportion in each category and representative examples. The 100 randomly evaluated rules and all rules identified by each method are available in supplementary material (Tables S1-S5).

Table 3. Taxonomy of drugs and associations in higher-order association rules

\begin{tabular}{|l|l|l|l|}
\hline \multicolumn{2}{|l|}{ Drugs } & AR & CARD \\
\hline 1 & Drug combinations known to interact & $10 \%$ & $20 \%$ \\
\hline 2 & $\begin{array}{l}\text { Drug combinations known to be given together or treat the same } \\
\text { disease }\end{array}$ & $4 \%$ & $13 \%$ \\
\hline 3 & Drug combinations that seem to be due to other confounding issues & $7 \%$ & $17 \%$ \\
\hline 4 & Drug combinations that are unknown to interact & $79 \%$ & $50 \%$ \\
\hline Associations & $\begin{array}{l}\text { One/more drugs in antecedent can cause the adverse event in } \\
\text { consequent }\end{array}$ & $61 \%$ & $77 \%$ \\
\hline a & No drug in antecedent can cause the adverse event in consequent & $39 \%$ & $23 \%$ \\
\hline
\end{tabular}

Table 4. Taxonomy of higher-order association rules

\begin{tabular}{|l|l|l|l|}
\hline Taxonomy & AR & CARD & Examples from CARD Findings \\
\hline $1-\mathrm{a}$ & $7 \%$ & $20 \%$ & (amiodarone + warfarin) $\rightarrow$ INR increased \\
\hline $2-\mathrm{a}$ & $1 \%$ & $10 \%$ & (alendronate + esomeprazole) $\rightarrow$ femur fracture \\
\hline $3-\mathrm{a}$ & $5 \%$ & $10 \%$ & (simvastatin + ramipril) $\rightarrow$ rhabdomyolysis \\
\hline $4-\mathrm{a}$ & $48 \%$ & $37 \%$ & (celecoxib + metformin) $\rightarrow$ myocardial infarction \\
\hline & & & \\
\hline $1-\mathrm{b}$ & $3 \%$ & $0 \%$ & (analgesics + cilostazol) $\rightarrow$ dyspnea (example from AR) \\
\hline $2-\mathrm{b}$ & $3 \%$ & $3 \%$ & (ranitidine + alendronate) $\rightarrow$ fall \\
\hline $3-\mathrm{b}$ & $2 \%$ & $7 \%$ & (aspirin + rosiglitazone) $\rightarrow$ coronary artery disease \\
\hline $4-\mathrm{b}$ & $31 \%$ & $13 \%$ & (acetaminophen + isotretinoin) $\rightarrow$ intestinal hemorrhage \\
\hline
\end{tabular}

Known drug interactions. Among the 100 randomly sampled findings, CARD identified more drug combinations known to interact compared to AR, $20 \%$ vs. $10 \%$. Further analysis suggested that 6 of the 20 CARD identified known interacting drug combinations cause the indicated AE 
(i.e., true positive DDI $\rightarrow$ AE associations). For example, CARD identified (amiodarone + warfarin) increases the international normalized ratio (INR) which is supported by evidence. This interaction is highly clinically significant and the risk of interaction often outweighs the benefit. As another example, (nitroglycerin + rosiglitazone) $\rightarrow$ myocardial infarction was identified by CARD and literature evidence also indicates that the combination should be avoided as it may increase the risk of myocardial ischemia.

The remaining 14 of the 20 interacting combinations identified by CARD are not currently known to cause the indicated $A E$ as an interaction effect but one or both drugs in the combination can still relate to the $\mathrm{AE}$ as known side-effects (i.e., Table 4 - taxonomy 1a). For example, the combination (prednisone + rosiglitazone) was identified to be associated with cardiac failure congestive. Existing evidence indicates that the combination have antagonistic effects where efficacy of the hypoglycemic agent, rosiglitazone, is decreased because prednisone can cause hyperglycemia. Thus cardiac failure congestive is not known to be a direct effect of the interaction; however it is a cardiovascular side-effect of prednisone due to long-term fluid retention and other direct vascular effects.

Another category of association for known interacting combinations is illustrated by the taxonomy $1 \mathrm{~b}$ where the identified AEs were unknown. CARD did not retrieve any of these pairs, but AR found 3. For example, prior clinical knowledge suggests that using cilostazol together with analgesics like aspirin may increase risk of bleeding, but not dyspnea as shown in Table 4.

Unknown drug interactions. CARD yielded a lower number of drug combinations unknown to interact, i.e., $50 \%$ for CARD vs. $79 \%$ for AR. Among the 50 false positives by CARD, 37 (74\%) are related to the identified $A E$ through one or both drugs (Table 4 - taxonomy 4-a). For instance, no evidence supports interaction between celecoxib and metformin, but the identified AE myocardial infarction is related to celecoxib. In contrast, 48 out of $79(61 \%)$ of the AR false positives are related to its identified $A E$. There are also unknown drug combinations with no connection to its identified AE. For example, there is no known interaction evidence between acetaminophen and isotretinoin and no information on either of the drugs causing intestinal hemorrhage.

Overlapping findings. There were only 4 drug combinations identified in common between CARD (Support $=100$, CAIM $>0$ ) and AR (Support $=100, R R \geq 2$ ), which is an unexpected but interesting finding. The four common drug combinations identified are (Table S5): (docetaxel, carboplatin), (pemetrexed, dexamethasone), (alendronate, docetaxel), and (dexamethasone, potassium). However, none of the pairs is indicated as interacting by Epocrates. Moreover, only 1 rule in common was found: (pemetrexed, dexamethasone) $\rightarrow$ pneumonia, but neither drug is 
known to be associated with pneumonia. The small number of overlapping is because AR and CARD have different preferences of the rules and different mining thresholds. In detail, CARD employs the CAIM to select the rule with threshold CAIM $>0$, while AR uses the RR to select the rule with $R R \geq 2$.

Confounded drug pairs. As shown in Tables 3 and 4, 30\% of CARD identified drug combinations are due to confounding factors such as concomitant (frequently co-administered medications) or indication (symptom of underlying disease, not effect of treatment) biases. As an illustration of taxonomy 2-a, alendronate was found to interact with esomeprazole to cause femur fracture. Alendronate is a bisphosphonate drug used for osteoporosis and other bone diseases. Esomeprazole is a proton pump inhibitor that reduces stomach acid secretion, used to treat dyspepsia, peptic ulcer disease, and gastroesophageal reflux disease. Some common adverse effects associated with alendronate include acid regurgitation and dyspepsia, which can be treated with esomeprazole, but the combination is not known to interact. This implies that our method may be finding $B N$ structures as depicted in Figure $2 a$ or $2 b$ rather than $2 d$, but conditional independence between the two medications can be wrongly estimated with missing $y$ node (indications of alendronate). Atypical femur fracture, identified as an AE for (alendronate + esomeprazole), is among the list of serious adverse reactions for alendronate and fracture is also one serious reaction of esomeprazole. Similarly for taxonomy 3-a, ramipril and simvastatin also seem to be a combination found due to confounding variables and not known to interact. The identified AE, rhabdomyolysis is simply a serious side-effect of simvastatin.

Furthermore for taxonomy 2-b, ranitidine is used to treat acid regurgitation and dyspepsia associated with alendronate, but not known to interact. In addition, neither drug is related to fall as indicated by CARD. Finally for taxonomy 3-b, aspirin and rosiglitazone also seems to be found due to confounding issues; aspirin should be a drug for treating coronary artery disease, not causing.

\section{Discussion}

Accurate identification of causal relationships between drug combinations and adverse events require not only analysis of all drug-event combinations but also cause-and-effect estimation of the relationships. Through the adoption of the essential properties of CBN V-structures in association rule mining, our proposed CARD method can efficiently identify causal associations between drug combinations and adverse events. A comparison to the traditional association rule 
mining method, CARD is demonstrated to identify more known drug interactions and yield a lower number of unknown drug combinations. Nonetheless, our study has limitations:

(1) CARD is not designed to identify causal relationships that cannot be represented with Vstructures. Theoretically in an ideal world, DDIs should form V-structures with its adverse effect. Thus, CARD is suitable for DDI discovery. In future work, we will explore other CBN structures and bootstrapping to identify persistently supported rules to increase confidence.

(2) Those identified rules unsupported by prior clinical knowledge could be spurious or warrant further pharmacologic analysis regarding mechanism of action. From an algorithm perspective, one not only needs to ensure precision on finding known associations is sufficiently high but also the method is able to discover new associations with high confidence. A potential approach to investigate the unknown associations in the future is to conduct retrospective studies using patient medical records.

(3) The overlapping finding between CARD and AR is low. One explanation is that the two algorithms theoretically favor different rule sets. AR prefers highly relevant rules measured by confidence and interestingness measures based on co-occurrence of itemsets and label. CARD conversely prefers item-sets with larger difference but higher predictability when combined, using mutual information as the penalty for discarding false rules generated from random combination of frequent item-sets. Another possible explanation for the little overlap may be the super large search space. For instance, our final dataset contained 7,701 drugs and only considering combination of 2 drugs would yield 29,648,850 pairs; hence CARD and AR may be identifying combinations from different parts of the actual DDI space.

This interesting observation made us more aware of the data granularity problem. Although free text drug names were codified to generic names with a NLP tool, it is not perfect; many written forms of drugs are not recognized and must be used in analysis as a unique concept. Furthermore, $A E$ granularity is a challenge as well $(11,569$ MedDRA coded AEs). Our future work needs to explore analyses with metacategorization of drugs and AEs by leveraging their hierarchical and ontological structures.

(4) Many of the CARD identified drug combinations are still due to confounding factors such as concomitant usage or indication biases. By design, CARD may identify more interrelated patterns than existing methods but can also inadvertently find more undesired confounded patterns. Since confounding variables correlate with both the dependent and independent variables, incorrect estimate of the relationship between variables may occur when confounding factors are unaccounted for. However, limited by 
the nature of the spontaneous reporting data, this study only considered drug events; missing essential phenotypic information such as indications and comorbidities. As our future work, a promising approach is to explore larger set of clinical variables from patient medical records standardized through our participation in PCORnet [51] and FDA's Sentinel initiative [52].

\section{Acknowledgements}

This work was partially funded through a number of research grants. R.C. was supported by the Natural Science Foundation of China $(61472089,61572143$, U1501254), the Natural Science Foundation of Guangdong Province, China (2014A030306004, 2014A030308008), Science and Technology Planning Project of Guangdong (2013B051000076, 2015B010108006, 2015B010131015) , Guangdong High-level personnel of special support program (2015TQ01X140) and Pearl River S\&T Nova Program of Guangzhou (201610010101) . Y.H. was supported by the National Science Foundation of China (71271061, 70801020), Science and Technology Planning Project of Guangdong Province, China (2010B010600034, 2012B091100192). M.E.M was supported by Veterans Administration HSR\&D Career Development Award CDA-08-020 and Veterans Administration HSR\&D Investigator Initiated Research IIR-11-292. H.X. was supported by NIGMS 1R01-GM103859, NLM 1R01LM011563, and CPRIT R1307. L.R.W was supported in part by the National Institutes of Health grant the Heartland Institute for Clinical and Translational Research (UL1TR000001).

\section{Author Contributions}

R.C., M.L. and Y.H. conceived the overall design, development, and evaluation of this study. M.L. and Y.H. prepared the dataset and designed the experiment for this study. R.C. implemented the method and conducted the experiments. M.E.M. and B.L.M. conducted the clinical evaluation of the results. H.X. contributed in the data preparation process by running MedEx for drug name mapping. L.D. and L.R.W. contributed in the writing of this manuscript. All authors reviewed the manuscript critically for scientific content, and all authors gave final approval of the manuscript for publication.

\section{Competing financial interests}

The authors declare no competing financial interests.

\section{References}


1. Health, United States 2014: with Special Feature on Adults Aged 55-64. (National Center for Health Statistics, Hyattsville, MD, 2015).

2. Lazarou, J., Pomeranz, B. H. \& Corey, P. N. Incidence of adverse drug reactions in hospitalized patients: a meta-analysis of prospective studies. JAMA 279, 1200-1205 (1998).

3. Johnson, J. A. \& Bootman, J. L. Drug-related morbidity and mortality. A cost-of-illness model. Arch Intern Med 155, 1949-1956 (1995).

4. Pirmohamed, M. Drug interactions of clinical importance. (Chapman \& Hall, 1998).

5. Khwaja, A. KDIGO clinical practice guidelines for acute kidney injury. Nephron. Clinical practice 120, c179-184 (2012).

6. Duke, J. D., Li, X. \& Dexter, P. Adherence to drug-drug interaction alerts in high-risk patients: a trial of context-enhanced alerting. J Am Med Inform Assoc 20, 494-498 (2013).

7. Banda J M, Callahan A, Winnenburg R, et al. Feasibility of prioritizing drug-drug-event associations found in electronic health records[J]. Drug safety 39(1): 45-57 (2016).

8. Goldman, J. L., Sullins, A., Sandritter, S., Leeder, J. S. \& Lowry, J. Pediatric Pharmacovigilance Enhancing Adverse Drug Reaction Reporting in a Tertiary Care Children's Hospital. Therapeutic Innovation and Regulatory Science 47, 566-571 (2013).

9. Bate, A. \& Evans, S. J. Quantitative signal detection using spontaneous ADR reporting. Pharmacoepidemiol Drug Saf 18, 427-436 (2009).

10. de Abajo, F. J., Montero, D., Madurga, M. \& Garcia Rodriguez, L. A. Acute and clinically relevant drug-induced liver injury: a population based case-control study. $\mathrm{Br} \mathrm{J}$ Clin Pharmacol 58, 71-80 (2004).

11. Almenoff, J. S., DuMouchel, W., Kindman, L. A., Yang, X. \& Fram, D. Disproportionality analysis using empirical Bayes data mining: a tool for the evaluation of drug interactions in the post-marketing setting. Pharmacoepidemiol Drug Saf 12, 517-521 (2003).

12. van Puijenbroek, E. P. et al. A comparison of measures of disproportionality for signal detection in spontaneous reporting systems for adverse drug reactions. Pharmacoepidemiol Drug Saf 11, 3-10 (2002).

13. Szarfman, A., Machado, S. G. \& O'Neill, R. T. Use of screening algorithms and computer systems to efficiently signal higher-than-expected combinations of drugs and events in the US FDA's spontaneous reports database. Drug Saf 25, 381-392 (2002).

14. Bate, A. et al. A Bayesian neural network method for adverse drug reaction signal generation. Eur J Clin Pharmacol 54, 315-321 (1998). 
15. Hauben, M., Madigan, D., Gerrits, C. M., Walsh, L. \& Van Puijenbroek, E. P. The role of data mining in pharmacovigilance. Expert Opin Drug Saf 4, 929-948 (2005).

16. Almenoff, J. et al. Perspectives on the use of data mining in pharmaco-vigilance. Drug Saf 28, 981-1007 (2005).

17. Almenoff, J. S. et al. Novel statistical tools for monitoring the safety of marketed drugs. Clin Pharmacol Ther 82, 157-166 (2007).

18. Ahmed, I., Thiessard, F., Miremont-Salame, G., Begaud, B. \& Tubert-Bitter, P. Pharmacovigilance data mining with methods based on false discovery rates: a comparative simulation study. Clin Pharmacol Ther 88, 492-498 (2010).

19. Van Puijenbroek, E. P., Egberts, A. C., Meyboom, R. H. \& Leufkens, H. G. Signalling possible drug-drug interactions in a spontaneous reporting system: delay of withdrawal bleeding during concomitant use of oral contraceptives and itraconazole. $\mathrm{Br} \mathrm{J}$ Clin Pharmacol 47, 689-693 (1999).

20. Thakrar, B. T., Grundschober, S. B. \& Doessegger, L. Detecting signals of drug-drug interactions in a spontaneous reports database. Br J Clin Pharmacol 64, 489-495 (2007).

21. van Puijenbroek, E. P., Egberts, A. C., Heerdink, E. R. \& Leufkens, H. G. Detecting drugdrug interactions using a database for spontaneous adverse drug reactions: an example with diuretics and non-steroidal anti-inflammatory drugs. Eur J Clin Pharmacol 56, 733-738 (2000).

22. Tatonetti, N. P. et al. Detecting drug interactions from adverse-event reports: interaction between paroxetine and pravastatin increases blood glucose levels. Clin Pharmacol Ther 90, 133-142 (2011).

23. Tatonetti, N. P., Fernald, G. H. \& Altman, R. B. A novel signal detection algorithm for identifying hidden drug-drug interactions in adverse event reports. J Am Med Inform Assoc 19, 79-85 (2012).

24. Harpaz, R., Chase, H. S. \& Friedman, C. Mining multi-item drug adverse effect associations in spontaneous reporting systems. BMC Bioinformatics 11 Suppl 9, S7 (2010).

25. Harpaz, R. et al. Biclustering of adverse drug events in the FDA's spontaneous reporting system. Clin Pharmacol Ther 89, 243-250 (2011).

26. Pearl, J. Causality: models, reasoning and inference. First edition edn, (Cambridge Univ Press, 2000). 
27. Schulz, K. F., Chalmers, I., Hayes, R. J. \& Altman, D. G. Empirical evidence of bias. Dimensions of methodological quality associated with estimates of treatment effects in controlled trials. JAMA 273, 408-412 (1995).

28. Rothman, K. J. Causes. 1976. American journal of epidemiology 141, 90-95; discussion 89 (1995).

29. Dekkers, O. M., von Elm, E., Algra, A., Romijn, J. A. \& Vandenbroucke, J. P. How to assess the external validity of therapeutic trials: a conceptual approach. International journal of epidemiology 39, 89-94 (2010).

30. Heckerman, D., Geiger, D. \& Chickering, D. M. Learning Bayesian networks: the combination of knowledge and statistical data. Machine Learning 20, 197-243 (1995).

31. Hoyer PO, Janzing D, Mooij J, Peters M, Scholkopf B. Nonlinear causal discovery with additive noise models. Neural Information Processing Systems (NIPS). 2008:689-96.

32. Zhang K, Hyvärinen A. On the identifiability of the post-nonlinear causal model. UAI. 2009: 647-655.

33. Pearl, J. From Bayesian network to causal networks. Bayesian Networks and Probabilistic Reasoning, 1-31 (1994).

34. Chickering, D. M., Heckerman, D. \& Meek, C. Large-sample learning of Bayesian networks is NP-hard. J Mach Learn Res 5, 1287-1330 (2004).

35. Cooper, G. F. A simple contraint-based algorithm for efficiently mining observational databases for causal relationships. Data Mining and Knowledge Discovery 1, 203-224 (1997).

36. Pellet, J. P. Using Markov blankets for causal structure learning. J Mach Learn Res 9, 1295-1342 (2008).

37. Aliferis, C. F., Statnikov, A., Tsannardinos, I., Mani, S. \& Koutsoukos, X. D. Local causal and Markov blanket induction for causal discovery and feature selection for classification Part I: Algorithms and empirical evaluation. J Mach Learn Res 11, 171-234 (2010).

38. Cai R, Zhang Z, Hao Z. Causal gene identification using combinatorial V-structure search. Neural Networks, 43: 63-71 (2013).

39. Cai R, Zhang Z, Hao Z. SADA: A General Framework to Support Robust Causation Discovery. ICML, 208-216 (2013).

40. Ji, Y. et al. A potential causal association mining algorithm for screening adverse drug reactions in postmarketing surveillance. IEEE transactions on information technology in 
biomedicine : a publication of the IEEE Engineering in Medicine and Biology Society 15, 428-437 (2011).

41. Li, J. et al. in IEEE 13th International Conference on Data Mining Workshops (ICDMW) 114-123 (Dallas, TX, 2013).

42. $\mathrm{Xu}, \mathrm{H}$. et al. MedEx: a medication information extraction system for clinical narratives. J Am Med Inform Assoc 17, 19-24 (2010).

43. Welcome to MedDRA and the MSSO, <http://www.meddramsso.com/MSSOWeb/ index.htm>

44. Noren, G. N., Orre, R., Bate, A. \& Edwards, I. R. Duplicate detection in adverse drug reaction surveillance. Data Mining and Knowledge Discovery 14, 305-328, doi:DOI 10.1007/ s10618-006-0052-8 (2007).

45. Reich, L. 'Extreme duplication' in the USFDA adverse events reporting system database. Drug Safety 30, 551-554 (2007).

46. Jin, H. W. et al. Mining unexpected temporal associations: applications in detecting adverse drug reactions. IEEE transactions on information technology in biomedicine, 488-500 (2008).

47. Vinh, N. X., Epps, J. \& Bailey, J. Information Theoretic Measures for Clusterings Comparison: Variants, Properties, Normalization and Correction for Chance. J Mach Learn Res 11, 2837-2854 (2010).

48. KDIGO. KDIGO Clinical Practice Guideline for Acute Kidney Injury. Kidney international. Supplement 2, 1-141 (2012).

49. Epocrates, <http://www.epocrates.com>

50. UpToDate, <http://www.uptodate.com>

51. Hou, S. H., Bushinsky, D. A., Wish, J. B., Cohen, J. J. \& Harrington, J. T. Hospital-acquired renal insufficiency: a prospective study. The American journal of medicine 74, 243-248 (1983).

52. McCullough, P. A. et al. Risk prediction of contrast-induced nephropathy. The American journal of cardiology 98, 27K-36K, doi:10.1016/j.amjcard.2006.01.022 (2006).

\section{APPENDIX}


Association rule mining is a well-researched method for discovering interesting relationships between variables in large databases. The relations discovered are typically in the form of rules $A \rightarrow B$, where $A$ and $B$ in this case denote a set of drugs and a set of adverse events, respectively (e.g., (aspirin, warfarin) $\rightarrow$ bleeding). A major limitation of the traditional association rule mining method is that the strength of rules is measured based on correlations, which does not imply causality. To address the limitation in this study, we introduce the concept of causality into association rule mining by proposing a Causal Bayesian network based method called Causal Association Rule Discovery (CARD). The CARD algorithm is described in detail as follows.

\section{CARD - Causal Association Rule Discovery}

\section{Problem Definition}

Let denote the indicators of the drugs taken, where is a binary indicator of the action whether a patient has taken the th drug or not. Let be a binary indicator of the occurrence of an adverse drug reaction (ADR) and represents an ADR report $j$. Let denote the event that ${ }^{\text {th }}$ drug is taken () and denote the event that an ADR has occurred (), then an adverse reaction triggered by drug-drug interaction (DDIs) can be formulated as an association rule, . As DDI involves more than one drug, we only focus on the rules with. Given a set of ADR reports, , we aim to discover drug combinations that cause adverse reactions through causal association rule discovery.

In the following sections, we first formalize the concept of causal association rules, then derive important properties of casual association rules, and finally propose an efficient causal association rule discovery method.

\section{Concept of Causal Association}

Causal faithfulness condition is a commonly used assumption in causal discovery problems. According to the causal faithfulness condition, there exists a causal Bayesian network faithful to the joint probability distribution defined on . For example, Figure 1 illustrates a causal Bayesian network with the drug and reaction variables as nodes. In the Bayesian network, each directed edge indicates the direct causal influence between the parent node and child node. Thus, in Figure 3 denote the direct causes of ADR $y$.

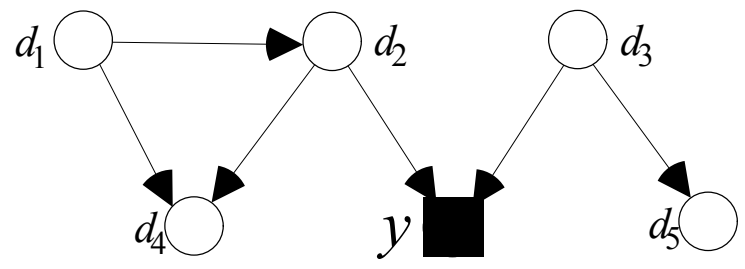


Figure 3. Example causal Bayesian network ( $d=$ drugs, $y=$ reaction)

Based on the direct causes, we can define the causal association rule as in Definition 1. The causal association rules in the form of are the focus of this study, where the corresponding variables and, and are all the direct causes of the ADR event A (i.e., $y=1$ ).

Definition 1. Causal Association Rule: an association rule, , defined on , is a causal association rule if are the direct causes of .

\section{Properties of Causal Association}

Although a lot of work has been conducted on the concept of causality, such as Dr. Judea Pearl's inductive causality method [1], Dr. Peter Spirtes' causal Bayesian network based methods [2], additive noise model [3], and a hybrid approach [4], causal discovery on high dimensional and sparse adverse drug-drug interaction data is still an open problem. Instead of reconstructing a causal Bayesian network, we propose to use the properties of causal Bayesian network to derive important properties of causal association rules, and apply such properties to guild the search of causal association rules.

In the simplest case, a causal Bayesian network with three variables can have four primitive structures as shown in Figure 2. The structures in Figure $2 a, 2 b$, and $2 c$ are independent-equivalent because they entail the same set of conditional independence relationships. In other words, variable is conditionally independent of variable given variable (i.e. ). However, Figure $2 \mathrm{~d}$ forms a V-structure and implies a different assertion that there exists a variable set and variable is conditionally independent of variable given (i.e. holds), but dependent given (i.e. does not hold).

As defined by Pearl [1],"two causal models are equivalent if and only if their direct acyclic graphs have the same links and the same set of uncoupled head-to-head nodes", in which the uncoupled head-to-head nodes indicate a V-structure. This suggests that causal associations can be inferred from $\mathrm{V}$-structures.

\section{Approach to Causal Association Rule Discovery}

Accordingly, we propose a causality interesting measure for the rule based on the properties of V-structure. Consider the simplest case, , if both and are causes of , then the corresponding three variables, and of , and forms a V-structure, . Thus, the following conditions must hold:

1. There does not exist satisfying or

2. There exists satisfying and. where can be an empty set. 
To measure the strength of association and independence relation, we use the normalized mutual information [5]. The above properties can be transferred to the following three heuristic rules of the causal association interestingness measure:

- $\quad$ and should be highly associated with, thus the rule with higher is preferred. Here is the normalized mutual information;

- and should be independent of each other given some variable set, thus the rule with lower is preferred;

- $\quad$ and should be dependent of each other given some variable set, thus the rule with higher is preferred

Combining the above three heuristic rules, we obtain the following causal association interestingness measure (CAIM):

For the rule with more than three antecedents, any sub-rules containing two antecedents must also form a V-structure with the adverse drug reaction, . Because the interestingness of rule is dependent on the weakness of its sub-rules, the generalized interestingness measure is defined as follows.

The interestingness defined in Formula (2) has a good monotonic property with the increasing number of antecedents (Theorem 1), which can be used in the mining procedure to accelerate interesting rule mining process. The proof of the theorem is straightforward and skipped here.

Theorem 1. Monotonic property: given any two association rules and, if , then holds.

Based on the definition of given in Formula (2), we can derive the following incremental updating strategy for CAIM. The incremental updating strategy provides an efficient way to estimate the interestingness when combined with incremental association rule mining approach.

Theorem 2. Incremental updating strategy for : given two association rules and an item , .

In addition to the interestingness measure, we can also derive the following pruning strategy of rules based on the properties of V-structures.

Theorem 3. Pruning strategy: given a causal association rule, there do not exist two variables satisfying, where, and are the corresponding variables of , and respectively.

Proof: Because is a causal association rule and, corresponding variables on and form a Vstructure. Based on the properties of V-structure, we know that does not hold.

Based on the CAIM, the proposed CARD algorithm is as follows. 


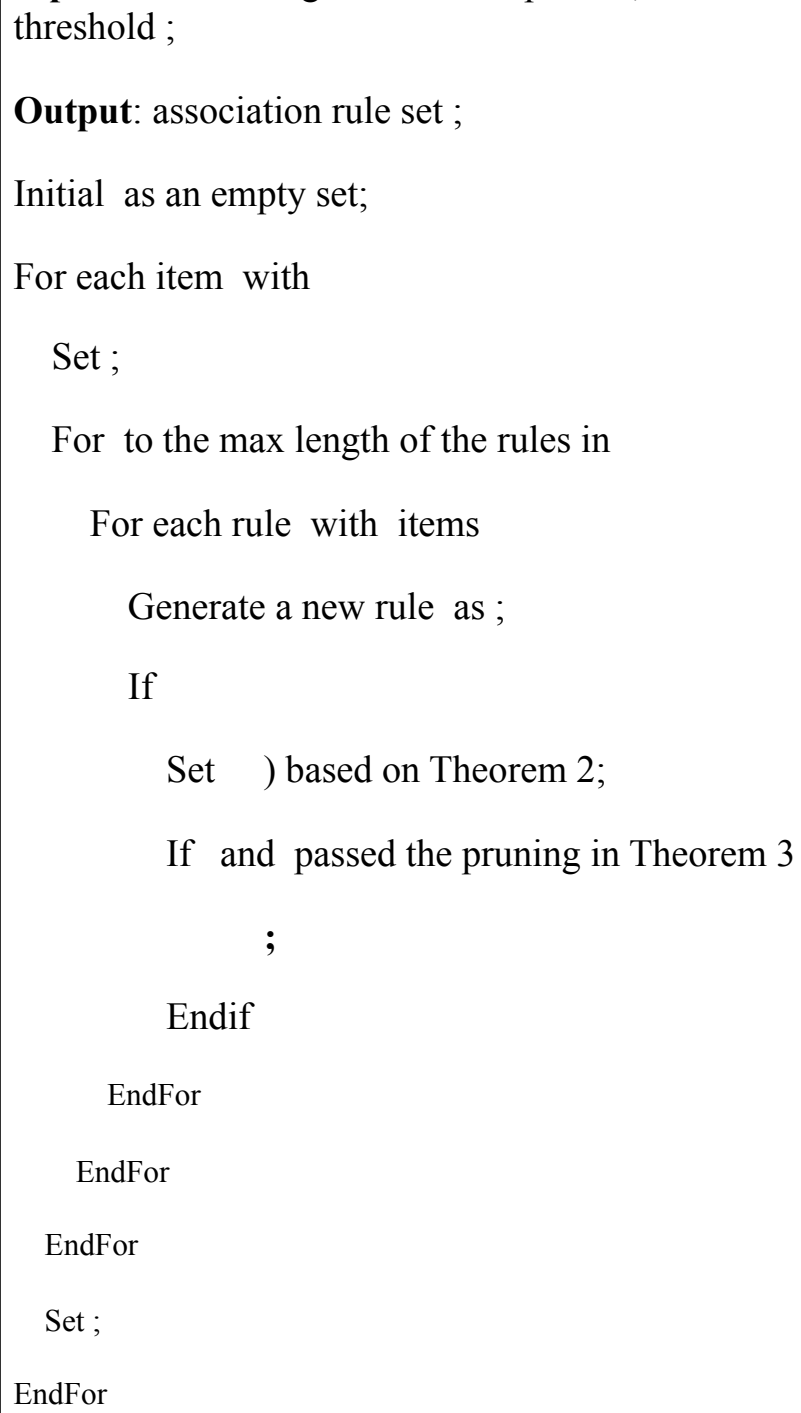

The algorithm shown above is adopted from the incremental rule mining algorithm proposed in [6]. It takes three input variables including the ADR sample set and two user specified parameters, minimal support threshold and minimal CAIM threshold, and returns a set of potential causal association rules by conducting a mixture of breadth-first and depth-first search in the association rule lattice space. More specifically, the items are sequentially added to the lattice maintained as rule set in a breadth-first manner. To process item, existing lattice is partitioned and processed in the depth manner, i.e. the item is added to the rules with 1 item, then the rules with 2 items, and so on and so forth. For each newly generated rule, the support and CAIM are calculated, only the ones that passed the support threshold, CAIM threshold, and the pruning process can be added to the rule set. More details about the incremental mining method can be found in [5]. 


\section{REFERENCE}

1. Pearl J. Causality: models, reasoning and inference. First edition ed: Cambridge Univ Press, 2000.

2. Spirtes P, Glymour C, Scheines R. Cusation, Prediction, and Search. Second Edition ed, 2001.

3. Hoyer PO, Janzing D, Mooij J, Peters M, Scholkopf B. Nonlinear causal discovery with additive noise models. Neural Information Processing Systems (NIPS). Vancouver, Canada, 2008:689-96.

4. Cai R, Zhang Z, Hao Z. Sada: A general framework to support robust causation discovery. Proceedings of the 30th International Conference on Machine Learning, 2013:208-16.

5. Vinh NX, Epps J, Bailey J. Information Theoretic Measures for Clusterings Comparison: Variants, Properties, Normalization and Correction for Chance. J Mach Learn Res 2010;11:2837-54

6. Cai R, Tung AKH, Zhang ZJ, Hao ZF. What is Unequal among the Equals? Ranking Equivalent Rules from Gene Expression Data. IEEE T Knowl Data En 2011;23(11):1735-47 